\title{
Itinerário terapêutico de idosas com lombalgia crônica
}

Itinerary of therapeutic elderly

chronic with low back pain

FisiSenectus. Unochapecó Ano 4, n. 1 - Jan/Jun. 2016 p. $12-21$

Luana Debiasi. Iuanadebiasi@unochapeco.edu.br

Fisioterapeuta Graduada na Universidade Comunitária da Região de Chapecó - Unochapecó.

Jaine Renata de Oliveira Zeni. jaine.zeeni@unochapeco.edu.br

Fisioterapeuta Graduada na Universidade Comunitária da Região de Chapecó - Unochapecó.

Ana Paula Maihack Gauer. anagauer@unochapeco.edu.br

Mestre em Ciências da Saúde pela Universidade Comunitária da Região de Chapecó Unochapecó.

Fátima Ferretti. ferrettifisio@yahoo.com.br

Doutora em Saúde Coletiva. Docente do Programa de Pós-Graduação Strictu Sensu em Ciências da Saúde, Universidade Comunitária da Região de Chapecó - Unochapecó.

\section{Resumo}

Introdução: Nas últimas décadas o Brasil envelheceu e a maior parte dessa população carrega consigo doenças próprias do envelhecimento. Dentre as doenças crônicas que mais acometem os idosos, destaca-se a lombalgia crônica, que é um quadro de dor que gera desconforto. Objetivo: Identificar o itinerário terapêutico de idosas com lombalgia crônica. Materiais e métodos: Pesquisa qualitativa que utilizou entrevista com questões norteadoras na coleta de dados. Foi realizada análise de conteúdo temática a partir de três etapas: pré-análise, exploração de material, tratamento dos resultados e interpretação. Resultados: Foram selecionadas 4 idosas com cognitivo preservado, média de idade de 63,5 anos e 8,75 anos de diagnóstico médico de lombalgia. Após análise, emergiram 5 categorias que detalharam o processo de busca por tratamento para a dor crônica lombar, desde os sintomas até a fase atual. Considerações finais: Conhecer o itinerário terapêutico de idosas com dor crônica lombar constituiu-se numa estratégia para ampliar as informações sobre as dificuldades enfrentados na busca pelo tratamento, e como estabelecer uma ação mais eficaz para a saúde destas pessoas.

\section{Palavras-chave}

Dor lombar; Idosos; Terapêutica. 


\begin{abstract}
Introduction: In recent decades, Brazil ages and most of this population carries with it own diseases of aging. Among chronic diseases that most affect the elderly, there is chronic low back pain, which is a painful condition that causes discomfort. Objective: The study aims to identify the therapeutic itinerary of elderly with chronic low back pain. Materials and methods: Qualitative research used the interview with guiding questions to collect data. The thematic content analysis from three pre-analysis steps, exploitation material, processing of results and interpretation. Results: 4 elderly women with preserved cognitive, mean age of 63.5 years and 8.75 years of medical diagnosis were selected. After analysis, five categories emerged that detailed the search process by treatment for chronic low back pain. Final considerations: Knowing the therapeutic itinerary of elderly with low back pain is chronic in a strategy to expand the information on difficulties experienced in the process and a way to reflect on the care offered, and how to establish a more effective action for the health of these people.
\end{abstract}

\title{
Keywords
}

Low back pain; The elderly; Therapeutics

\section{Introdução}

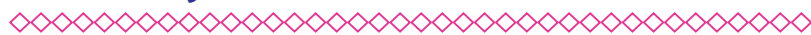

Nas últimas décadas o Brasil envelheceu em grande proporção e o número de idosos aumentou em quase $700 \%$ em menos de 50 anos, passando de 3 milhões, em 1960, para 7 milhões, em 1975, e 20 milhões em 2008. A maior parte desses idosos carrega consigo doenças próprias do envelhecimento. Dentre elas, destacam-se as doenças crônicas, que perduram por anos, com exigência de cuidados constantes, medicação contínua e exames periódicos ${ }^{1}$. 0 envelhecimento pode produzir alterações que aumentam os fatores de risco e a ocorrência de doenças crônico-degenerativas² ${ }^{2}$.

Dentre as enfermidades crônicas que acometem as mulheres idosas, uma das mais comuns é a dor lombar, principalmente no período pós-menopausa, já que nessa fase há uma alteração na composição corporal, com maior presença de gordura e menor massa magra, o que pode contribuir para a intensidade dos quadros álgicos ${ }^{3}$. A dor crônica se destaca entre os principais fatores que diminuem o índice de qualidade de vida em idosos e nível de independência nas atividades de vida diária (AVD's) 4 .

Em pesquisa realizada para identificar a prevalência e características da dor crônica de idosos numa grande metrópole, observou-se prevalência de $29,7 \%$ de dor crônica, sendo a região lombar o local com maior frequência de acometimento ${ }^{5}$. Em função da gravidade do quadro e do desconforto gerado pela dor, esses sujeitos percorrem caminhos distintos durante a busca por tratamento, resultando no que chamamos de itinerário terapêutico. Entende-se por itinerário terapêutico o trajeto que o usuário percorreu, desde a descoberta da patologia, todas as tentativas de tratamento até o estágio em que se encontra6.

Conhecer o caminho percorrido pelas idosas com lombalgia crônica na busca pelo tratamento possibilita, aos profissionais da área da saúde, o reconhecimento de estratégias de cuidados que podem ser incentivadas e acrescidas no tratamento destes pacientes, transformando a atenção oferecida numa ação mais eficaz para a saúde. Considerando o contexto apresentado e a necessidade de se conhecer essa realidade, este estudo teve como objetivo identificar o itinerário terapêutico de idosas com lombalgia crônica atendidas numa Clínica Escola de Fisioterapia de Santa Catarina.

\section{Materiais e métodos}

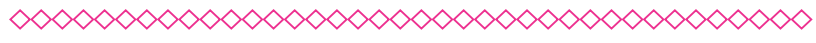

0 presente estudo é de caráter qualitativo. Este se ocupa de um nível de realidade que não pode ser quantificado, ou seja, trabalha com um universo de significados, de crenças, de valores e de atitudes, caracterizando-se como um conjunto de fenômenos humanos entendidos como parte da realidade social. Esta abordagem justifica-se quando há a intenção de aprofundar a investigação no mundo das ações e relações humanas, se preocupa com 
um nível da realidade social, cujos processos e fenômenos não podem ser quantificados ou reduzidos à operacionalização de variáveis ${ }^{7}$.

A seleção das idosas deu-se de modo intencional. Os critérios estabelecidos para a seleção foram: ser idosas de 60 a 70 anos, ter realizado tratamento fisioterapêutico na clínica escola nos últimos 5 anos ou estar em tratamento, possuir diagnóstico médico de lombalgia no prontuário e referir quadro álgico há mais de 6 meses, possuir pontuação equivalente a cognitivo preservado, conforme MEEM, e atender a ligação telefônica de contato, entre 1 e 3 tentativas.

Inicialmente, foi realizado contato com a Clínica Escola de Fisioterapia, informando sobre a pesquisa, com vistas a receber autorização para sua realização. Após receber aceite, o projeto foi submetido na Plataforma Brasil para apreciação ética. Na sequência, realizou-se a busca pelas idosas nos prontuários dos pacientes atendidos na Clínica Escola de Fisioterapia, conforme critérios de seleção preestabelecidos. Posteriormente, realizou-se contato telefônico para agendar a entrevista no domicílio em data e horários definidos pelas idosas.

Após ter acesso aos prontuários da clínica e aplicar os critérios de seleção foram incluídas 12 idosas no estudo. Destas, 4 não atenderam o telefone após três tentativas e 4 realizaram tratamento na Clínica Escola para outra finalidade que não a dor lombar, estando a queixa de dor crônica lombar secundária no plano de tratamento, o que resultou em 4 sujeitos potenciais para o estudo.

Na primeira visita ao domicílio das idosas apresentou-se o projeto de pesquisa, seus objetivos e intencionalidade. Aqueles que aceitaram participar assinaram o Termo de Consentimento Livre e Esclarecido. Para iniciar as coletas foi aplicado o Mini Exame do Estado Mental (MEEM) que é aplicado para avaliar a função cognitiva em vários domínios (orientação espacial, temporal, memória imediata e de evocação, cálculo, linguagem-nomeação, repetição, compreensão, escrita e cópia de desenho), considerado de fácil aplicação por ser rápido e por não requerer material específico que apresenta com o objetivo a condição cognitiva.

Em seguida foi aplicada entrevista com questões norteadoras relacionadas ao diagnóstico, percurso de tratamentos, motivos de adesão ao tratamento, facilidades e dificuldades para acessar o tratamento em função da lombalgia crônica. A entrevista, de acordo com Minayo ${ }^{7}$, pode ser caracterizada por uma conversa entre duas ou mais pessoas, realizada por iniciativa do entrevistador, destinada a construir informações pertinentes para um objeto de pesquisa. As entrevistas foram gravadas e transcritas, posteriormente, foi solicitada a validação das mesmas na íntegra.

Foi realizada Análise de Conteúdo Temática, que ocorre em três etapas: Pré-análise, que consiste na escolha dos documentos a serem analisados e na retomada das hipóteses e dos objetivos iniciais da pesquisa, reformulando-as frente ao material coletado, bem como na elaboração de indicadores que orientem a interpretação final; Exploração do Material que consiste na operação de codificação; Tratamento dos Resultados Obtidos e Interpretação, onde os resultados brutos são submetidos a operações estatísticas simples ou complexas que permitem colocar em relevo as informações obtidas ${ }^{7}$.

Este estudo garantiu os princípios éticos no desenvolvimento de atividade com seres humanos respeitando as Diretrizes e Normas da Resolução 466/CNS/2012, do Conselho Nacional de Saúde. A proposta de investigação foi submetida à apreciação e aprovação do comitê de ética da Unochapecó, sob o parecer n. 1.318.285.

\section{Resultados e discussões}

$\infty \times \infty \times \infty \times \infty \times \infty \times \infty \times \infty \times \infty \times \infty \times \infty \times \infty \times \infty$

Foram selecionadas 4 idosas com média de idade de 63,5 anos e 8,75 anos em média de diagnóstico médico de lombalgia. Todas as entrevistadas eram casadas, do lar e alfabetizadas. Todas apresentaram cognitivo preservado conforme Mini Exame de Estado Mental (MEEM), com pontuação máxima igual ou superior a 24 pontos para pessoas alfabetizadas.

Conhecer o itinerário terapêutico consiste em uma ferramenta de valor para a compreensão de determinados comportamentos em relação ao processo saúde-doença-cuidado do usuário. Tal conhecimento possibilita aprimorar o funcionamento dos serviços, no que diz respeito à qualificação da assistência, levando à construção de 
propostas de intervenção que sejam centradas no indivíduo e que possam considerar seu contexto social e história de vida a fim de atingir resultados terapêuticos mais efetivos.

A interpretação do material empírico oriundo das entrevistas, realizada por meio de análise de conteúdo temática, foi organizada em categorias e subcategorias que detalham o processo de busca por tratamento para a dor crônica lombar relatada pelos sujeitos do estudo, que inicia com o aparecimento dos sintomas.

\section{Sintomas iniciais da lombalgia}

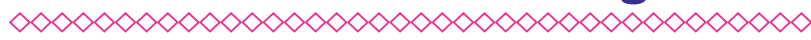

Em relação aos sintomas iniciais da lombalgia as entrevistadas relataram a dor:

"A vida toda senti essas dores nas costas. Em algum período melhorava, após atendimento na unidade básica de saúde e ganhava alguns remédios e acalmava um pouco, em alguns dias voltava de novo..." (Sujeito 1, 68 anos).

"Eu sentia dores terríveis nos braços e na coluna, eu não conseguia nem mexer muito o pescoço. Tinha muitos torcicolos e muitas dores nas pernas [...]. Eu fui tomando analgésico, colocando compressa quente, quando eu não aguentava mais usava um remédio [...]" (Sujeito 4, 64 anos).

"Eu sentia muita, muita dor nas costas, parecia uma queimação na coluna..." (Sujeito 1, 68 anos).

"Sempre senti muita dor nas costas, e eu trabaIhava, então sentia muita dor nos ombros e na coluna." (Sujeito 3, 61 anos).

Os relatos das idosas indicam que o primeiro sintoma foi a dor. 0 estudo que verificou a prevalência de dor lombar em idosos evidenciou que $62 \%$ dos pesquisados referiram dor lombar, e destes, mais da metade, 52,9\% referiram dor lombar de intensidade moderada, evidenciando que a dor, quando cotidiana, traz prejuízos importantes para a saúde física e psíquica destes e, ainda, altos custos de tratamento ${ }^{8}$

Os episódios de dor, como no caso das lombalgias, acarretam dificuldades para o idoso realizar tarefas do dia a dia, que, normalmente, são indispensáveis para uma vida independente. A dor, como sintoma inicial, que levou as idosas a buscar o tratamento, esteve relacionada com a limitação para desenvolver as atividades rotineiras, e sua intensidade levou as idosas a procurar recursos na área da saúde para minimizar o quadro ${ }^{9}$. 0 mesmo constatou-se no presente estudo, em que a dor lombar intensa diminuiu as atividades de vida diária das idosas e foi precursora da busca por tratamento para cessá-la, dando início ao percurso realizado pelas idosas na busca pela melhoria das condições de saúde.

Para além da dor isolada, outro sintoma que levou as idosas a buscar tratamento foi o estresse decorrente da dor, como demonstrado nos relatos abaixo:

"Eu ficava muito nervosa por conta da dor, tive muitas câimbras e necessitei ficar parada." (Sujeito 1, 68 anos)

"Fico estressada, tenho sentido muitas dores, para mim as dores são uma das piores coisas da vida, pois me limitam para tudo, para fazer uma comida, um artesanato ou para sair." (Sujeito 4, 64 anos).

"Ficava muito irritada, porque eu sentia muita dor e tinha que ir trabalhar." (Sujeito 3, 61 anos).

As queixas de nervosismo, estresse e ansiedade são comuns na população idosa que sofre com dor crônica. Nos primeiros momentos, com a presença da dor, a busca por tratamento pode ser motivada pela expectativa de se obter um diagnóstico e uma terapêutica que diminuam o sofrimento. Os indivíduos parecem optar por utilizar estratégias ativas de analgesia e não depender de profissionais ${ }^{5}$. Assim, os pacientes acabam buscando ajuda profissional somente em casos de extrema necessidade, quando não suportam mais conviver com tal incomodo, e neste instante, buscam ajuda especializada.

Esse fato pode ser considerado um ponto negativo no processo de diminuição dos sintomas da dor, uma vez que, adiada a busca por tratamento, como com o surgimento do estresse, os agravos podem ser ainda mais intensos, retardando a eficácia da terapêutica, bem como, degradando o bem-estar referido e implementando o trajeto de tratamento traçado. 


\section{Entraves/dificuldades para acessar 0 tratamento de dor lombar \\ $\infty \times \infty \times \infty \times \infty \times \infty \times \infty \times \infty \times \infty \times \infty \times \infty \times \infty \times \infty \times \infty$}

Quanto às dificuldades e entraves mencionados pelos sujeitos de pesquisa para acessar ao tratamento, destaca-se o custo elevado, que está evidenciado nos relatos abaixo:

"A dor começou no trabalho ainda, iniciei a hidroginástica... Nem sei se retorno, houve um aumento alto da mensalidade." (Sujeito 4, 64 anos).

"Também tomei muito suplemento vitamínico, mas é muito caro pra comprar..." (Sujeito 1, 68 anos).

"Essa cirurgia que fiz foi paga por mim, alguns exames demoram e outros eu paguei, como a ressonância, por exemplo, que demorou uns dois anos e então, [...] fomos direto ao laboratório." (Sujeito 2, 61 anos).

As falas das idosas detalham o custo elevado com tratamentos, para além do oferecido no serviço, o que dificulta o acesso integral de cuidados. Apesar do fácil acesso aos serviços ofertados pelo SUS, há aspectos limitantes nesse meio, que fazem os usuários buscar por tratamento particular. Estudo de Mehret ${ }^{10}$ encontrou achados semelhantes, pois observou que a lombalgia exige tratamento complexo, já que se trata de uma síndrome multicausal, e, muitas vezes, o custo de tratamento é alto para a camada da população de renda baixa. Isso faz com que os pacientes acabem não realizando o tratamento completo ou abandonem, podendo ser percebível na subcategoria a seguir.

Outra dificuldade elencada pelas idosas, foi a demora para atendimento e realização de procedimentos e exames, evidenciado nas seguintes falas:

"O problema é a demora e a espera. Espera para sair exame, espera para marcar consulta, tudo! Às vezes você vai lá pra agendar consulta, tá morrendo de dor e leva um mês para conseguir. A maioria dos exames eu paguei, mas alguns consegui pelo SUS." (Sujeito 1, 68 anos).

"Sempre muita demora, meu Deus! Essa é uma dificuldade do postinho... A ressonância foi a que mais demorou, eu acho que demorou uns 8 meses naquela época." (Sujeito 3, 61 anos).
Estudo de Sousa e colaboradores ${ }^{11}$ que teve como objetivo analisar as condições para o acesso integral à rede assistencial, evidenciou que a população vem enfrentando obstáculos para acessar os serviços de saúde, desde o primeiro contato com a Unidade de Saúde e durante todo o caminho percorrido para a continuidade do cuidado, seja para exames, seja para atenção especializada. Dentre os obstáculos, um dos principais é a demora no agendamento das consultas especializadas e exames complementares.

Essa realidade está associada a grande demanda por serviços e número reduzido de profissionais e equipes na Atenção Básica, além da ausência de comunicação entre os serviços de diferentes níveis assistenciais, o que dificulta ainda mais a conduta terapêutica, fazendo com que as idosas busquem por novas terapêuticas, ampliando, assim, o itinerário dos pacientes com doenças crônicas.

\section{Facilidades para acessar o tratamen- to de dor lombar}

$\infty \times \infty \times \infty \times \infty \times \infty \times \infty \times \infty \times \infty \times \infty \times \infty \times \infty \times \infty \times \infty$

Dentre as facilidades que as idosas encontraram para acessar o tratamento, uma delas contrapõe uma dificuldade enunciada acima, o acesso aos exames e medicamentos pelo SUS, destacados nos seguintes relatos:

"Era tudo particular menos os exames que nunca paguei... fiz todo tipo de exame, até exame da cabeça, também recebia os medicamentos para dor..." (Sujeito 1, 68 anos).

"Fiz bastante exame pelo SUS, exame da coluna, ressonância, raio-x. Fiz um monte de coisa... Foi na ressonância que diagnosticaram, e foi tudo pelo SUS..." (Sujeito 3, 61 anos).

"Só tomo quando sinto muita dor, paracetamol ou nimesulida que eles dão..." (Sujeito 2, 61 anos).

As idosas que participaram do estudo descrevem que o acesso a exames e medicamentos pelo SUS são facilidades vivenciadas na busca pelo tratamento para a lombalgia crônica. Estudo realizado com 131 usuários de saúde que receberam cuidados em infarto agudo do miocárdio, alcoolismo, câncer de mama e parto, observou que o índice de 
satisfação em relação aos prestadores de serviços é alto ${ }^{12}$, como evidenciado nas falas.

Estudo realizado com 24 usuários do SUS, em que se buscou analisar a percepção dos usuários sobre as facilidades de acesso às ações e aos serviços do SUS, demonstrou uma atitude positiva em relação ao fácil acesso a diversos tipos de serviços, incluindo consultas, exames, medicações ou serviços de alta complexidade ${ }^{13}$.

Outro item que veio à tona no percurso percorrido pelas idosas na busca do tratamento foi 0 atendimento humanizado evidenciado nas seguintes falas:

"Tem bastante fila, mas os médicos são bem bons e dão bastante atenção, inclusive quando o médico do postinho me encaminhou para o especialista..." (Sujeito 3, 61 anos).

"E depois da fisioterapia melhorei muito... Foi a mão que eu precisava mesmo, não em medicamento, pois eu não queria medicamento, eu precisava daquela atenção que os idosos e as pessoas mais velhas precisam..." (Sujeito 4, 64 anos).

Para Waldow e Borges ${ }^{14}$ humanizar pode ser considerado uma forma de cuidado integral. Já para Silva e Silveira ${ }^{15}$, a humanização faz-se necessária no sentido de qualificar o atendimento e conscientizar o profissional da saúde, para que reconheça o paciente como um sujeito e não como um simples objeto de sua atenção.

Como o exposto acima, o atendimento humanizado mostra-se como a união da ética com conhecimento e, evidencia, muitas vezes, o laço afetivo que os idosos criam com os profissionais, 0 que ocorre com pacientes idosos, que em algumas situações estão carentes de atenção. Esse aspecto pode interferir no processo de adesão ao tratamento pelos idosos, nos casos de lombalgia crônica, visto que é um paciente que está fragilizado pelo quadro álgico, o que requer um atendimento diferenciado.

Outra facilidade destacada pelos idosos, no percurso realizado em busca do tratamento, foi possuir o plano de saúde complementar, o que implica na percepção dos idosos em atendimento com mais brevidade, conforme relato das entrevistadas:
"Eu tenho plano de saúde para complementar, e também acho que tenho sorte, pois ontem eu estava com dor no joelho, eu liguei lá e já consegui consulta para a mesma tarde." (Sujeito 4, 64 anos).

"Quando o exame é mais caro eu preciso pagar, tem muitas outras coisas que também preciso pagar. E com meu plano, só pago a metade das coisas..." (Sujeito 3, 61 anos).

Os idosos adotam uma relação intensa com os serviços de saúde, principalmente por se tratar de uma população frágil, com várias demandas, levando-os, muitas vezes, a aderir a planos de saúde complementares, uma vez que o SUS pode traduzir inadequações que dependem de políticas públicas integradas para se efetivar ${ }^{16}$. O Brasil hoje é um jovem país de cabelos brancos. A cada ano, 650 mil novos idosos são incorporados à população brasileira, a maior parte com doenças crônicas e alguns com limitações funcionais. Em menos de 40 anos, o Brasil passou de um cenário de mortalidade próprio de uma população jovem para um quadro de enfermidades complexas e onerosas, típica dos países longevos, caracterizado por doenças crônicas e múltiplas que perduram por anos, com exigência de cuidados constantes, medicação contínua e exames periódicos ${ }^{1}$, o que sobrecarrega o sistema de saúde. Nessa direção, a adesão, por parte do idoso, a planos de saúde complementares, na busca por tratamento com o objetivo de alcançar o sucesso e rapidez nos serviços ofertados, passa a ser percebido pelos idosos como um aspecto facilitador no caminho terapêutico traçado.

\section{Tratamentos realizados}

$\infty \times \infty \times \infty \times \infty \times \infty \times \infty \times \infty \times \infty \times \infty \times \infty \times \infty \times \infty \times \infty)$

Dentre os tratamentos realizados, todas utilizaram medicamentosos para aliviar a dor, conforme relatos a seguir:

“Tomei bastante remédio: diclofenaco, paracetamol, injeção voltaren e também sempre tive remédio para dor por perto." (Sujeito 1, 68 anos).

"E remédio para dor tomo direto até hoje." (Sujeito 3, 61 anos).

"Eu fiquei cheia de problema na coluna. E aí então fui tomando analgésico e colocando compressa quente daí, quando eu não aguentava mais, fui ao médico." (Sujeito 4, 64 anos). 
Com o avançar da idade, as pessoas acabam desenvolvendo doenças crônicas e acabam utilizando uma grande quantidade de medicamentos, os quais podem trazer benefícios quando usados corretamente pelo paciente, e podem trazer prejuízos se não utilizados adequadamente ${ }^{17}$. A população idosa caracteriza-se por possuir diversos problemas crônicos de saúde e utilizar vários medicamentos, sendo na sua maioria polimedicados. A adoção da automedicação é uma prática descrita dentro do autocuidado, sendo adotada pela grande maioria dos idosos para o manejo de sintomas, em especial a dor ${ }^{18}$. 0 fato da automedicação fica claro no perfil apresentado: idosas com dor crônica lombar, reiterando a facilidade e os riscos da prática da polimedicação nessa população.

Como se percebeu nos relatos acima, o principal efeito esperado pelas idosas, ao aderir ao uso dos medicamentos, analgésicos e relaxantes, era diminuir a dor, garantir o relaxamento e bem-estar.

Outro recurso utilizado na busca pelo tratamento foi a massagem corporal, trazida como muito eficaz pelos relatos a seguir:

"Fiz massagem na Dona E.." (Sujeito 1, 68 anos).

"Já fiz bastante massagem e me ajudou muito, fiz também relaxamentos." (Sujeito 2, 61 anos).

Estudo sobre os benefícios da massoterapia no tratamento de paciente com fibromialgia demonstrou que a massoterapia é eficaz na diminuição dos sintomas e no limiar de dor ${ }^{19}$. Os efeitos da massoterapia ou massagem corporal também foram relatados pelas idosas no presente estudo como um coadjuvante eficaz no tratamento da dor lombar crônica, proporcionando melhora na percepção e bem-estar referido. A busca por diferentes recursos no processo de tratamento favorece a adesão e soma positivamente na vida dos sujeitos.

A reabilitação fisioterapêutica foi considerada eficaz durante o processo de melhoria da dor crônica lombar, conforme evidenciada nos relatos:

"Fisioterapia então, já perdi as contas de tantos lugares que fiz. Sempre ajuda." (Sujeito 1 , 68 anos).

"Já fiz bastante fisioterapia, e o que sempre resolveu para mim é atividade, atividade, atividade! Alongamento e musculação. Eu aprendi muito lá, para mim não foi uma mão foram as duas mãos..." (Sujeito 4, 64 anos).
“O doutor falou que eu não poderia parar nunca a fisioterapia, e isso está ajudando bastante, meu Deus! Fazendo exercício diminui a dor, o doutor também indicou pilates, mas eu faço na clínica com as estagiárias e me alongo em casa..." (Sujeito 2, 61 anos).

0 tratamento fisioterapêutico contribui para a melhora da autoestima das idosas, conduzindo-as a sentirem-se mais seguras, independentes, respeitadas, reconhecidas e adequadas à vida ${ }^{20}$. Além disso, proporciono sensação de bem-estar e revigoramento com melhora da elasticidade dos músculos. A maioria dos idosos reconhece a importância da fisioterapia em suas vidas, e percebem que ela traz algo bom e melhora a sua saúde. Há que se manter o idoso ativo e independente, pelo maior período de tempo, o que melhora sua imagem, autoestima e saúde ${ }^{21}$.

Estudo sobre a satisfação de idosos em atendimento numa clínica universitária quanto ao tratamento realizado, demonstrou alta satisfação dos usuários, sendo que boa parte contava com a possibilidade de realizar os atendimentos em instituições privadas, mas optavam por dar sequência em seus tratamentos na clínica-escola22.

O tratamento fisioterapêutico, ou a prática de exercícios físicos, proporciona melhor desempenho funcional, como observado nesse estudo, no qual os idosos portadores de dor crônica lombar destacaram a reabilitação fisioterapêutica como imprescindível no processo de adesão ao tratamento, tornando-se um diferencial no caminho percorrido.

Houve, também, relatos sobre o tratamento fitoterápico, e o que este auxiliou durante o trajeto, evidenciados a seguir:

"Chá eu tomo. Na situação que me encontro não tem como não tomar e faz bem!". (Sujeito 2,61 anos).

“Chá eu tomo até hoje, até ontem de noite fiz um para tomar porque não aguentava mais de dor." (Sujeito 3, 61 anos).

Estudo de Silva et al. ${ }^{23}$ com 94 idosos evidenciou que a utilização de plantas medicinais é uma prática comum entre esses sujeitos, sendo que $90,4 \%$ dos idosos faziam o uso, desses, $85,9 \%$ eram do gênero feminino. Vários fatores, como a questão cultural quanto ao uso dos chás, o saber 
repassado de mãe para filha, o cuidado associado à figura feminina, podem se constituir em fatores que interferem nesse achado, no maior uso de chás pelas mulheres.

Os sujeitos do estudo atual relataram que a utilização de plantas medicinais é um importante recurso na busca por tratamento, e que pode estar ligado à cultura e à tradição de cada uma, e foi considerado um diferencial do itinerário terapêutico dessas idosas.

\section{Situação atual}

$\infty<\infty<\infty<\infty<\infty<\infty<\infty<\infty<\infty<\infty<\infty<\infty<\infty<\infty<\infty<\infty<$

Por fim, quando perguntadas sobre a situação atual em que se encontram, tendo em vista o trajeto percorrido na busca por tratamento da dor lombar crônica, as entrevistadas discorreram:

"A fisioterapia me ajudou muito, antes eu não varria e nem passava pano na casa, agora eu me viro, estou bem melhor." (Sujeito 1, 68 anos).

"E depois que iniciei na fisioterapia a dor diminuiu bastante." (Sujeito 2, 61 anos).

Muitos idosos sentem dor e convivem com ela diariamente, tentando manter uma vida normal, muitos por medo de serem mal interpretados, tachados de poliqueixosos, ou, ainda, já terem se acostumado com a não valorização de sua dor. Alguns idosos, mesmo com dor, não deixam de manter um convívio social, familiar, com amigos, com grupos, o que evidencia uma boa capacidade de adaptação, quando o quadro álgico é minimizado. Redes de suporte social contribuem para que o indivíduo acredite que são cuidados, amados e valorizados, sentindo-se pertencente ao grupo em que possam dar e receber apoio emocional. Os relacionamentos sociais são muito importantes para todas as pessoas, também para as idosas com dor crônica ${ }^{24}$.

A dor crônica é um quadro que pode ser controlado, mas que se não tiver tratamento adequado, interfere significativamente na condição de saúde e vida da pessoa idosa. Para garantir melhores condições de saúde, as idosas desse estudo buscaram diferentes terapias para a amenização dos sintomas; os medicamentos, a Fisioterapia, o pilates, as complementares, entre outras.

Conhecer o itinerário terapêutico utilizado pelas idosas com lombalgia põe em evidência as dificuldades vivenciadas pelos pacientes na busca pelo cuidado, advindas na descoberta da doença e na procura de atendimento nas redes de atenção e de cuidado à saúde. Muitas vezes, essas se exacerbam com as limitações impostas pela idade e pela doença, levando-as ao sofrimento individual e familiar. Nessa perspectiva, buscam diferentes estratégias e formas de superar essa fase, que compõe o itinerário terapêutico destas mulheres.

\section{Considerações finais}

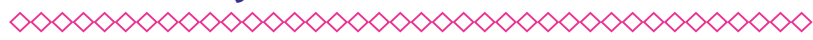

A descoberta dos primeiros sintomas da dor crônica lombar se deu em diferentes momentos: durante as atividades laborais, amparo a familiares debilitados e afazeres domésticos.

0 estudo aponta as dificuldades enfrentadas pelas idosas durante o percurso realizado em busca do tratamento. Destacam-se o custo elevado do tratamento e a demora nos atendimentos e realização de procedimentos e exames. Como facilidades na busca por tratamento, destacou-se o acesso aos exames pelo SUS, atendimento humanizado e plano de saúde complementar. Ressalta-se, também, as crenças religiosas na reabilitação como ferramenta para adesão ao tratamento realizado. Dentre os tratamentos realizados para sanar a dor, foram elencados: tratamento medicamentoso, massagem corporal, reabilitação fisioterapêutica e tratamento fitoterápico.

Finalizando, ressalta-se que conhecer os itinerários terapêuticos destas idosas com dor crônica lombar constituiu-se numa estratégia para melhor compreender os processos de saúde e adoecimento e a construção das relações de cuidados no tempo e no espaço. Essas informações podem promover o adensamento em torno do debate quanto ao desenvolvimento de linhas de cuidados, com abordagens mais integralizadoras e humanizadas, que fortaleçam os sujeitos frente ao processo de cuidado e garantam o direito à saúde. 


\section{Referências}

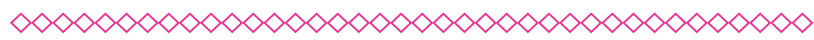

1. Veras R. Envelhecimento populacional contemporâneo: demandas, desafios e inovações. Rev Saúde Pública. 2009;43(3):548-54.

2. Torres GV, Reis LA, Reis LA, Fernandes $M H$, Alves GS. Avaliação da capacidade de realização das atividades cotidianas em idosos residentes em domicílio. Rev Baiana Saúde Pública. 2009;33(3):466-75.

3. Maraschin R, Vieira PS, Leguisamo CP, Dal'Vesco F, Santi JP. Dor lombar crônica e dor nos membros inferiores em idosas: etiologia em revisão. Fisioter Mov. (Impr.). 2010 Dez;23(4):627-639.

4. Barbosa MH, Silva LC, Andrade EV, Luiz RB, Bolina AF, Mattia AL, et al. Avaliação da dor crônica em idosos institucionalizados. Rev Min Enferm. 2012;16(1):63-8.

5. Dellaroza MSG, Pimenta CAM, Lebrão ML, Duarte YA. Associação de dor crônica com uso de serviços de saúde em idosos residentes em São Paulo. Rev Saúde Pública. 2013;47(5):914-22.

6. Oliveira BRG, Collet N, Mello DF, Lima RAG. O itinerário terapêutico de famílias de crianças com doenças respiratórias no sistema público de saúde. Rev Latino-Am Enfermagem. 2012 Jun;20(3):453-461.

7. Minayo MCS. O Desafio do Conhecimento: pesquisa qualitativa em saúde. 14a ed. São Paulo: Hucitec; 2014.

8. Figueiredo VF, Pereira LSM, Ferreira PH, Pereira AM, Amorim JSC. Incapacidade funcional, sintomas depressivos e dor lombar em idosos. Fisioter Mov. 2013 Set;26(3):549-557.

9. Mascarenhas CHM, Santos LS. Avaliação da dor e da capacidade funcional em indivíduos com lombalgia crônica. Health Sci Inst. 2011;29(3):205-8.

10. Mehret MOC, Colombo CCG, Lopes SS. Estudo comparativo entre as técnicas de a acupuntura auricular, craneoacupuntura de yamamoto, eletroacupintura e cinesioterapia no tratamento da Iombalgia crônica. Rev Bras Terap e Saúde. 2010;1(1):1-12.

11. Sousa FOS, Medeiros KR, Gurgel Júnior GD, Albuquerque $P C$. Do normativo à realidade do Sistema Único de Saúde: revelando barreiras de acesso na rede de cuidados assistenciais. Ciência \& Saúde Coletiva. 2014;19(4):1283-93.

12. Sisson MC, Oliveira MC, Conill EM, Pires D, Boing AF, Fertonani HP. Satisfação dos usuários na utilização de serviços públicos e privados de saúde em itinerários terapêuticos no sul do Brasil. Interface (Botucatu). 2011 Mar;15(36):123-136.

13. Pontes APM, Cesso RGD, Oliveira DC, Gomes AMT. Facilidades de acesso reveladas pelos usuários do Sistema Único de Saúde. Rev Bras Enferm. 2010 Ago;63(4):574-580.

14. Waldow VR, Borges RF. Cuidar e humanizar: relações e significados. Acta Paul. Enferm. 2011;24(3):414-8.

15. Silva ID da, Silveira M de FA. A humanização e a formação do profissional em fisioterapia. Ciência \& Saúde Coletiva. 2011;16(11):1535-46.

16. Louvison MCP, Lebrão ML, Duarte YAO, Santos JLF, Malik AM, Almeida E S. Desigualdades no uso e acesso aos serviços de saúde entre idosos do município de São Paulo. Rev Saúde Pública. 2008 Ago;42(4):733-740.

17. Pinto NBF, Vieira LB, Pereira FMV, Reis AMM, Cassiani SHB. Interações medicamentosas em prescrições de idosos hipertensos: prevalência e significância clínica. Rev Enferm UERJ. 2014;22(6):735-41.

18. Cascaes EA, Falchetti ML, Galato D. Perfil da automedicação em idosos participantes de grupos da terceira idade de uma cidade do sul do Brasil. Arquivos Catarinenses de Medicina. 2008;37(1).

19. Silva M da; Silva MP da; Macedo AB. Os benefícios da massoterapia no tratamento de paciente com fibromialgia - um estudo de caso. Revista Eletrônica "Saúde CESUC". 2010;1(1).

20. Kunz RI, Silva JR, Coradini JG, Tavares KO, Frare JC. Autoestima e satisfação com a vida 
na perspectiva de idosas inseridas em um programa de exercícios físicos. Revista UNINGÁ. 2014;17(3):11-7.

21. Banzatto S, Alves AGRC, Silva CM, Viana MO, Freitas IMP, Menezes JNR. Análise da efetividade da fisioterapia através da psicomotricidade em idosos institucionalizados. Rev Bras Promoç Saúde. 2015;28(1):119-25.

22. Santos MB, Ferraz RRN, Dias TOS, Neri ASC, Oliveira RS de. Satisfação dos idosos com relação ao atendimento fisioterapêutico ambulatorial como instrumento de gestão em uma clínica universitária. Revista de Administração de Roraima. 2014;4(2):4-14.

23. Silva, Allan Batista, Araújo CRF, Mariz SR, Meneses AB, Coutinho MS, Alves RBS. 0 uso de plantas medicinais por idosos usuários de uma unidade básica de saúde da família. Rev Enferm. 2015;9(3):7636-43.

24. Celich KLS, Galon C. Dor crônica em idosos e sua influência nas atividades da vida diária e convivência social. Rev Bras Geriatr Gerontol. 2009;12(3):345-59. 